\title{
Studying the Status of Job Burnout and its Relationship with Demographic Characteristics of Nurses in Shiraz Nemazee Hospital
}

\author{
Tahereh Shafaghat ${ }^{1}$, Mohammad-Kazem Rahimi-Zarchi ${ }^{1,}$, Zahra Kavosi ${ }^{1}$ \\ ${ }^{1}$ Department of Health Services Management, School of Management and Medical Informatics, Shiraz University of Medical Sciences, \\ Shiraz, Iran
}

*Corresponding Author: Mohammad-Kazem Rahimi-Zarchi, Ph.D. Candidate in Health Services Management, Department of Health Services Management, School of Management and Medical Informatics, Shiraz University of Medical Sciences, Shiraz, Iran. Tel: +98-9132529084; Email: Rahimi_1399@yahoo.com

Received: 5 Oct. 2015; Accepted: 24 Nov. 2015; Online Publish: 11 Jan. 2016

\begin{abstract}
Background: Today, in order to provide desirable health care services, too much emphasis is placed on the physical and mental health of nurses, and job burnout among nurses is introduced as harmful elements to the health of nurses.

Objective: This study was performed to evaluate job burnout in Shiraz Nemazee Hospital in relation to demographic characteristics.

Methods: This research is a cross-sectional and descriptive-analytic study. The research community included all nurses in the whole sections of Nemazee Hospital out of which 245 were selected by classified random sampling as the study sample. A questionnaire was used to collect the data. After collection, data were entered in statistical package for social sciences SPSS software (version 18) and T-test, and analysis of variance (ANOVA) and Kruskal-Wallis tests were used to analyze the variables.

Results: The mean score of emotional exhaustion, lack of personal accomplishment and job burnout were at an average level, and depersonalization was at a low level. As regards the intensity of burnout, most nurses were moderate. Between components of depersonalization of job burnout with marital status and age, there was a significant relationship $(\mathrm{P}<0.05)$. Also, nurses in neurological wards were allocated the most $(62.28 \%)$ while nurses in children ward recorded the lowest (49.92\%) mean of burnout.

Conclusion: According to the findings of this study and in terms of the stressful nature of nursing profession, it is necessary that hospital managers and healthcare authorities pay attention to job burnout in nurses, its level, as well as provide and implement strategies for its prevention, thereby decreasing its effects and risks.
\end{abstract}

Keywords: Burnout, Nurses, Hospitals

\section{Background}

Today, in order to provide optimal healthcare services, much emphasis is placed on the physical and mental health of nurses. Also, job burnout has been introduced as a factor which affects the health of nurses [1]. Studies have shown that one of the reasons responsible for job burnout is job stress and there are some evidence that nursing is a stressful profession $[1,2]$.

In the nursing profession, factors such as shifts, high working pressure, conflicts with colleagues, experiencing the sufferings and deaths of patients, professional responsibilities and issues relating to office systems, constitute major occupational stress [3]. On the long run, occupational stresses lead to job burnout [4].

Job burnout have numerous complications in the family, social, personal and organization life including absenteeism and leave serving, successive delays, various psychological complaints, conflicts, change of jobs and reduced quality of care given to patients, lack of care towards patients and interpersonal conflicts with co-workers, just to mention a few $[5,6]$.

In researches conducted by Nastiezaie et al. and BahriBinabaj et al., the job burnout level was reported moderately in a desired society, and significant correlation was seen between job burnout and general health [7, 8]. In a study by Mohammadi, there was a significant correlation between job burnout and general health [9].

Anisi et al. in their studies observed a significant relationship between demographic characteristics and job burnout [10].

\section{Objective}

In this study, an evaluation of job burnout level in nurses working in Shiraz Nemazee Hospital, in 2013 was carried out, and the effect of factors, such as age, gender, marital status, job experience, education level, and job ward on burnout was determined.

\section{Methods}

This research is a practical, cross-sectional and descriptive-analytic study. The research community included all nurses working in whole wards of Shiraz Nemazee Hospital. The sampling method in this research was random stratified sampling in proportion with the number of nurses in each ward of Nemazee Hospital.

First, each hospital ward was considered as a stratum, then the total sample size $(n=245)$ which corresponded to the number of nurses in each hospital ward was divided. Afterwards, in proportion with the number of nurses in each ward, some nurses of each ward were randomly selected and verbally satisfied to complete the questionnaire.

The used questionnaire consisted of 2 parts: demographic data and job burnout questionnaire. According to previous researches, the job burnout questionnaire has a high validity and reliability. The reliability and validity were calculated for each burnout dimension and Cronbach's alpha was 
reported as: emotional exhaustion (90\%), depersonalization (79\%), individual performance $(71 \%)$ and questionnaire reliability generally was announced as between 71 to $90 \%$ [11].

The questionnaire contained 22 items in 3 dimensions. The emotional exhaustion dimension consisted of 9 questions, depersonalization dimension consisted of 5 questions, and lack of personal accomplishment consisted of 8 questions. The score of each question was a variable from 0 to 6 . The total scores of questions of each dimension represented each person's score in that dimension. The acquisition of further scores represented more problems.

For this questionnaire, two types of scales were used:

Frequency scale: never (zero), several times in a year (1), once a month (2), several times a month (3), once a week (4), several times a week (5), and every day (6).

Severity scale: never (zero), very low (1) Low (2), medium (3), top medium (4), high (5), and very high (6).

Scores obtained were interpreted in this way:

The emotional exhaustion (low: between 0 to 18 score, medium: score between 19 to 36, high: score between 37 to 54)

Depersonalization (low: score between 0 to 10 , medium: score between 11 to 20, high: score between 21 to 30 )

Lack of personal accomplishment (low: the score 0 to 16 , medium: score between 17 to 32 , high: a score between 33 to 48 )

Job burnout (low: score between 0 to 44 , medium: score between 45 to 88 , high: score between 89 to 132 [7].

The demographic data questionnaire also included variables such as: age, gender, marital status, education level, work experience and type of work ward.

After studying and checking questionnaires and entering data in the SPSS software (version 18), the descriptive statistics was used to review and analyze the descriptive variables while T-test, ANOVA and Kruskal-Wallis statistically tests were used to analyze the analytical variables. Statistical ANOVA test was used to determine the relationship between ages, work experience and education level with job burnout level. T-test was used to determine the relationship between gender and marital status with job burnout level, and to determine the significance of differences in job burnout level in various wards. Also, the non-normality of nurses' distribution in various wards, was assessed using the Kruskal-Wallis test.

\section{Results}

The majority of nurses in Shiraz Nemazee hospital were women $(86.5 \%)$ who mostly were in the age group between 20 and 30 years $(57.1 \%)$, married $(62.4 \%)$, have work experience between 1 to 5 years $(51.8 \%)$, have bachelor degree and more (85.3\%), and working in the surgery ward (34.3\%) (Table 1).

A majority of the nurses, in terms of emotional exhaustion, lack of personal accomplishment and total job burnout, were located at the medium level, while in terms of depersonalization they were at a low level (Table 2). Therefore, in all work wards, the intensity of job burnout was medium. Also, neurological ward nurses were allocated the highest $(62.28 \%)$ mean while children ward nurses recorded the lowest (49.92\%) mean of job burnout (Table $3)$.

There was no significant relationship between any dimension of job burnout and the various job wards (p>0.05) (Table 4).
Between depersonalization dimension with age and marital status, there was a significant relationship $(\mathrm{p}<0.05)$. So that depersonalization in the age group 41 to 50 years and singles, was more than the other group. According to Table 5, between the dimensions of job burnout with gender, education level and work experience, there was no significant relationship $(\mathrm{p}<0.05)$ (Table 5).

Table 1. Frequency of nurses based on demographic characteristics

\begin{tabular}{lcc}
\hline Demographic Specification & n $(\%)$ \\
\hline \multirow{2}{*}{ Age } & $20-30$ years & $140(57.1)$ \\
& $31-40$ years & $80(32.7)$ \\
Gender & $41-50$ years & $25(10.2)$ \\
Marital status & Man & $33(13.5)$ \\
& Woman & $212(86.5)$ \\
Job experience & Married & $153(62.4)$ \\
& Single & $92(37.6)$ \\
& $1-5$ years & $127(51.8)$ \\
Education level & $6-10$ years & $59(24.1)$ \\
& $11-15$ years & $30(12.2)$ \\
& More than 15years & $29(11.8)$ \\
& Diploma & $21(8.6)$ \\
& Post diploma & $15(6.1)$ \\
Job ward & BS and more & $209(85.3)$ \\
& Neurology & $14(5.7)$ \\
& Internal & $15(6.1)$ \\
& Surgery & $84(34.3)$ \\
& Emergency & $14(5.7)$ \\
\hline & Children & $56(22.9)$ \\
& ICU & $28(11.4)$ \\
& Para clinic & $17(6.9)$ \\
& Operating room & $17(6.9)$ \\
\hline
\end{tabular}

Table 2. Frequency of intensity of job burnout and its components among nurses based on severity

\begin{tabular}{lccc}
\hline \multirow{2}{*}{ Variable } & Low & Medium & High \\
\cline { 2 - 4 } Emotional & $\mathbf{n}(\%)$ & $\mathbf{n}(\%)$ & $\mathbf{n}(\%)$ \\
\hline $\begin{array}{l}\text { exhaustion } \\
\text { Depersonalization }\end{array}$ & $108(44.1)$ & $112(45.7)$ & $25(10.2)$ \\
$\begin{array}{l}\text { Lack of personal } \\
\text { accomplishment }\end{array}$ & $15(62.9)$ & $88(35.9)$ & $3(1.2)$ \\
Job burnout & $60(10.6)$ & $183(74.7)$ & $36(14.7)$ \\
\hline
\end{tabular}

Table 3. Frequency of intensity of job burnout and its components among nurses based on job ward

\begin{tabular}{|c|c|c|c|}
\hline \multirow{2}{*}{$\begin{array}{l}\text { Intensity of Job } \\
\text { Burnout Job Ward }\end{array}$} & Low & Medium & High \\
\hline & n $(\%)$ & n (\%) & $\mathrm{n}(\%)$ \\
\hline Neurology & $1(7.1)$ & $11(78.6)$ & $2(14.3)$ \\
\hline Internal & $5(33.3)$ & $10(66.7)$ & $0(0)$ \\
\hline Surgery & $19(22.6)$ & $64(76.2)$ & $1(1.2)$ \\
\hline Emergency & $2(14.3)$ & $12(85.7)$ & $0(0)$ \\
\hline Children & $13(23.2)$ & $43(76.8)$ & $0(0)$ \\
\hline ICU & $11(39.3)$ & $17(60.7)$ & $0(0)$ \\
\hline Para clinic & $6(35.3)$ & $11(64.7)$ & $0(0)$ \\
\hline Operating room & $3(17.6)$ & $13(76.5)$ & $1(5.9)$ \\
\hline Total & $60(24.5)$ & $181(73.9)$ & $4(1.6)$ \\
\hline
\end{tabular}

\section{Discussion}

In terms of intensity of job burnout, $24.5 \%$ of nurses were in low, $73.9 \%$ were in moderate and $1.6 \%$ were in a high level. According to Dick, job Burnout is the severe exhaustion and feeling of being abused, such that this type of fatigue cannot be relieved by rest or even vacation.

$\mathrm{He}$ also believed that job burnout is a state that a person sees himself less than others and feels his job and action is not considered [12], therefore it must be said that $75.5 \%$ of the studied nurses experienced such problems. In his study, Bahri-Binabaj et al. arrived at the result that most midwives and nurses (78.2) at Gonabad University of Medical Sciences were at the medium level, in terms of job burnout [8].

Also, the results of the research by Kouranian et al. indicated that the majority of nurses $(79.3 \%)$ in terms of job burnout were at a medium level [13]. 
Table 4. Determination of the relationship between job burnout and its components with job wards

\begin{tabular}{|c|c|c|c|c|c|c|c|c|}
\hline \multirow{2}{*}{ Job Wards } & \multicolumn{2}{|c|}{ Job Burnout } & \multicolumn{2}{|c|}{ Emotional Exhaustion } & \multicolumn{2}{|c|}{ Depersonalization } & \multicolumn{2}{|c|}{ Lack of Personal Accomplishment } \\
\hline & Mean & Frequency & Mean & Frequency & Mean & Frequency & Mean & Frequency \\
\hline Neurology & 137.61 & 14 & 134.04 & 14 & 101.71 & 14 & 153.46 & 14 \\
\hline Internal & 108.77 & 15 & 118 & 15 & 134.87 & 15 & 86.20 & 15 \\
\hline Surgery & 130.06 & 84 & 135.42 & 84 & 134.43 & 84 & 115.20 & 84 \\
\hline ICU & 160.18 & 14 & 153.54 & 14 & 148.21 & 14 & 119.11 & 14 \\
\hline Emergency & 111.48 & 56 & 101.10 & 56 & 111.14 & 56 & 133.79 & 56 \\
\hline Children & 102.73 & 28 & 112.21 & 28 & 104.36 & 28 & 120.61 & 28 \\
\hline Para clinic & 106.82 & 17 & 110.38 & 17 & 111.41 & 17 & 113.88 & 17 \\
\hline Operating room & 145.53 & 17 & 134.32 & 17 & 134.18 & 17 & 149.50 & 17 \\
\hline P Value & \multicolumn{2}{|c|}{0.092} & \multicolumn{2}{|c|}{0.083} & \multicolumn{2}{|c|}{0.185} & \multicolumn{2}{|c|}{0.109} \\
\hline
\end{tabular}

Table 5. Determination of the relationship between job burnout and its components with demographic characteristics of nurses

\begin{tabular}{|c|c|c|c|c|c|c|c|c|c|}
\hline \multirow{2}{*}{\multicolumn{2}{|c|}{$\begin{array}{l}\text { Demographic } \\
\text { Characteristics }\end{array}$}} & \multicolumn{2}{|c|}{ Job Burnout } & \multicolumn{2}{|c|}{ Emotional Exhaustion } & \multicolumn{2}{|c|}{ Depersonalization } & \multicolumn{2}{|c|}{$\begin{array}{l}\text { Lack of Personal } \\
\text { Accomplishment }\end{array}$} \\
\hline & & Mean \pm SD & P Value & Mean \pm SD & P Value & Mean \pm SD & P Value & Mean \pm SD & P Value \\
\hline \multirow{3}{*}{ Age } & $20-30$ years & $54.633 \pm 16.78$ & \multirow{3}{*}{0.499} & $21.62 \pm 11.38$ & \multirow{3}{*}{0.411} & $9.22 \pm 5.26$ & & $23.77 \pm 6.98$ & \multirow{3}{*}{0.298} \\
\hline & $31-40$ years & $52.92 \pm 15.23$ & & $19.73 \pm 11.10$ & & $7.72 \pm 5.14$ & 0.045 & $25.46 \pm 8.83$ & \\
\hline & 41-50 years & $57.08 \pm 14.34$ & & $22.36 \pm 11.25$ & & $10.22 \pm 5.70$ & & $24.40 \pm 7.65$ & \\
\hline \multirow{2}{*}{ Gender } & Man & $54.27 \pm 14.69$ & \multirow{2}{*}{0.475} & $20.6 \pm 10.6$ & \multirow{2}{*}{0.831} & $8.9 \pm 4.92$ & & $24.75 \pm 6.91$ & \multirow{2}{*}{0.513} \\
\hline & Woman & $54.33 \pm 16.27$ & & $21.16 \pm 11.39$ & & $8.83 \pm 5.38$ & 0.295 & $24.75 \pm 6.91$ & \\
\hline Marital & Married & $54.86 \pm 14.42$ & \multirow{2}{*}{0.161} & $21.67 \pm 11.04$ & \multirow{2}{*}{0.203} & $8.83 \pm 4.77$ & 0033 & $24.35 \pm 6.52$ & \multirow{2}{*}{0.165} \\
\hline Status & Single & $54 \pm 16.97$ & & $20.74 \pm 11.43$ & & $8.85 \pm 5.63$ & 0.033 & $21.41 \pm 8.35$ & \\
\hline \multirow{4}{*}{$\begin{array}{l}\text { Job } \\
\text { experience }\end{array}$} & $1-5$ years & $53.92 \pm 15.44$ & \multirow{4}{*}{0.622} & $20.92 \pm 11.07$ & \multirow{4}{*}{0.766} & $8.80 \pm 5.08$ & & $24.19 \pm 6.44$ & \multirow{4}{*}{0.602} \\
\hline & $6-10$ years & $55.44 \pm 17.63$ & & $21.69 \pm 11.70$ & & $9.55 \pm 5.22$ & & $24.18 \pm 7.65$ & \\
\hline & $11-15$ years & $56.50 \pm 13.50$ & & $22.20 \pm 11.72$ & & $8.13 \pm 6.02$ & 0.604 & $34.34 \pm 6.85$ & \\
\hline & $\begin{array}{c}\text { More than } 15 \\
\text { years }\end{array}$ & $51.55 \pm 17.88$ & & $18.27 \pm 11.14$ & & $8.34 \pm 5.85$ & & $23.82 \pm 11.24$ & \\
\hline \multirow{3}{*}{$\begin{array}{l}\text { Education } \\
\text { level }\end{array}$} & Diploma & $54.76 \pm 22.98$ & \multirow{3}{*}{0.176} & $20.66 \pm 15.47$ & \multirow{3}{*}{0.100} & $7.8 \pm 6.47$ & & $26.28 \pm 9.35$ & \multirow{3}{*}{0.452} \\
\hline & Post diploma & $61.73 \pm 15.79$ & & $27.13 \pm 11.61$ & & $11.26 \pm 5.04$ & 0.140 & $23.23 \pm 5.19$ & \\
\hline & BS and more & $53.75 \pm 15.16$ & & $20.69 \pm 10.68$ & & $8.77 \pm 5.18$ & & $24.27 \pm 7.67$ & \\
\hline
\end{tabular}

Also, in a research by Barrett, on nurses from hematology and oncology wards, more than $70 \%$ of samples showed medium to high levels of job burnout [11]. Also, the research results of Adriaenssens et al. were somewhat affiliated with this study [1]. The difference between these statistics and numbers can be due to differences in studied populations and their age range. Rahmani's research indicated high levels of job burnout in nurses [14]. Also, the result of investigations of Lopez Franco and Anisi reflected a low level of job burnout [10, 15], and these results are inconsistent with the results of the present investigation.

In terms of emotional exhaustion, $44.1 \%$ of nurses were assessed to be at low level, $45.7 \%$ at medium and $10.2 \%$ at high level. According to the definition of Maslach and Jackson, emotional exhaustion is "a sense of compression and loss of a person's emotional resources". On one hand, emotional exhaustion leads to the loss of individual emotional energy and makes that person lose his motivation to act and do something boring, and when symptoms of emotional exhaustion appears, the person has the feeling of helplessness [16], and since nurses are exposed to numerous stresses due to the nature of their job [1], the work environment eventually drains the emotional and psychological resources, thus leading to burnout syndrome [17]. In other words, due to nurses' exposure to stressful situations, death of patients, and also being responsible for the care of patients with special conditions, they are faced with emotional exhaustion and their ability to support patients and give quality care is reduced. So, empowering nurses to cope with stressful factors alongside providing conditions for reduction and elimination of these factors can create a relaxed working environment and increase the capacity and efficiency of nurses [18] and reduce their level of emotional exhaustion.

In terms of depersonalization, $62.9 \%$ of nurses were at a low level, $35.9 \%$ at medium level and $1.2 \%$ at high level. This burnout dimension is related to non-emotional and violent responses of person on clients and partners. A person with job burnout syndrome has negative emotions and attitudes, blames others and sees people as objects and not humans [11]; for example, doctors with burnout see their patients as an object and identify them with a kind of disease, room number etc., and no longer feel the pains of the patient.

Also, in terms of lack of personal accomplishment, 10.6\% of nurses were observed to be at low level, $74.7 \%$ in medium and $14.7 \%$ at high level. In this dimension of job burnout, a person feels his performance is without success and in other words cognitive performance or success is important rather than the real success and performance [16].

Rahmani, in his study, discovered that the emotional exhaustion of nurses in the lung ward was high (45.8) [14]. Although, according to a research by Nastiezaie, more study subjects $(90.5 \%)$ showed depersonalization at low level [7]. Also, according to the study by Lopez Franco, $67.7 \%$ of nurses showed lack of personal accomplishment at low level, $14.5 \%$ showed high level of emotional exhaustion and $23.9 \%$ showed depersonalization at high level [15]. The study by Adriaenssens concluded that $27 \%$ of nurses showed lack of personal accomplishment, $26 \%$ showed emotional exhaustion and $35 \%$ showed depersonalization at a high level [1]. In the study by Canadas-De la Fuente et al., it was found that $41 \%$ of nurses had low level of emotional exhaustion, $39 \%$ of nurses had low level of depersonalization and $45 \%$ of them also had low level of lack of personal accomplishment [19]. Also, the study by Rafii showed that $39.5 \%$ of nurses had experienced lack of personal accomplishment at high level, $47 \%$ showed emotional exhaustion at low level and $75 \%$ experienced depersonalization at a high level [20].

In his study, Frade assessed emotional exhaustion and 
lack of personal accomplishment at low level and the depersonalization at high level; he also measured the severity of job burnout at $14 \%$ [21]. Santana Cabrera found high levels of emotional exhaustion and depersonalization in all groups [22].

Also, neurological ward nurses were allocated the highest value (62.28) while children ward nurses had the least (48.92) mean of job burnout. It seems that the special status of patients in the neurology wards, including restricted movements and the severity of pain experienced by patients in this ward influenced the nurses. On the other hand, perhaps the presence of children in the children ward cheered up the nursing staff and reduced job burnout.

Based on the research findings, there was a significant relationship between the depersonalization dimension of job burnout with age $(\mathrm{p}<0.05)$. Also, the highest mean score of depersonalization was in the age group of 41 to 50 years and the lowest was in the age group of 31 to 40 years. In explaining this, perhaps people attained the level of intellectual maturity between 31 to 40 years and in proportion to younger people, the experience of nurses increased in dealing with occupational issues. Therefore, the intensity of depersonalization and burnout reduced. However, in the age group of 41 to 50 years, perhaps, people because of low mood, fatigue, daily job and depression associated with this age group, will have depersonalization, take offence on people and offer cold or senseless treatment to others. Adali suggested that mild-life crisis enhanced a person's sensitivity to depression due to an increase in age, resulting in improved experience on the comprehension of burnout, thereby decreasing the effect [23]. In this regard, Rafii in his study concluded that there was a significant relationship between age and depersonalization $(\mathrm{P}<0.05) \quad$ [20]. Also, Anisi and Adriaenssens concluded that there was a significant relation and inverse correlation between age and job burnout [1, 10]. Thus, the results of these researches are approximately aligned with this study.

According to the study findings, there was no significant relationship between any of the job burnout dimensions with gender $(\mathrm{P}>0.05)$. Also, according to Lopez Franco's research, the demographic characteristics had the least involvement in job burnout [15] which was aligned with the current research. But in the research by Rafii and Adriaenssens, the existence of relationship between gender with all or some of burnout's components was shown [1, 20]; Although, Canadas-De la Fuente in his own study these differences which have been seen in some studies but not in others [19].

According to the findings of this study, there was only a significant relationship between the depersonalization dimension with marital status $(\mathrm{P}<0.05)$ and the mean of depersonalization in married people was more than single people. Perhaps, the reason for this was the problems associated with the married people, due to adding marital life problems and the burden of family responsibility to work problems and issues, and thus the depersonalization and job burnout was more in this people. Also, Anisi concluded that marital status did not have any significant relationship with job burnout [10].

\section{Conclusion}

According to the research findings and in terms of the stressful nature of nursing job, it is necessary that hospital managers and healthcare authorities pay attention to the effect of job burnout factor of nurses, its level, so as to provide solutions in order to avoid or at least decrease its effects and disadvantages. Some implications could focus on the promotion of adequate professional autonomy, creating time- out facilities and provision of counseling for exposed nurses and training nurses with coping skills.

\section{Acknowledgments}

We thank all nurses who cooperated with us on this research. It should be noted that this paper is the result of a research project that was approved by the Student Research Committee of Shiraz University of Medical Sciences with approval number "90-5863".

\section{Authors' Contributions}

All authors have equal role in this paper.

\section{Conflict of Interest}

No conflict of interest has been declared by the author(s).

\section{References}

1. Adriaenssens J, De Gucht V, Maes S. Determinants and prevalence of burnout in emergency nurses: A systematic review of 25 years of research. Int J Nurs Stud. 2015;52(2):649-61. [DOI]

2. Lee I, Wang H-H. Perceived occupational stress and related factors in public health nurses. J Nurs Res. 2002;10(4):253-60. [DOI]

3. Roberts R, Golding J, Towell T, Weinreb I. The effects of economic circumstances on British students' mental and physical health. J Am Coll Health. 1999;48(3):103-9. [DOI]

4. Embriaco N, Papazian L, Kentish-Barnes N, Pochard F, Azoulay E. Burnout syndrome among critical care healthcare workers. Curr Opin Crit Care. 2007;13(5):482-8. [DOI]

5. Gorrell B, Langenbach $M$. Curriculum issues related to nurse turnover. J Nurs Educ. 1994;33(3):101-6.

6. Hakim Shooshtari M, Khajeholdin N, Arabgol F, Ellahi F. The relationship between nurse burnout and their infant behavior problems. Adv Cogn Sci. 2004;6(1-2):67-72.

7. Nastiezaie N, Vaezi M, Molazahi A, Moghadam M. The relationship between job burnout and public health of telecommunication central office personnel in Zahedan. Toloo-eBehdasht. 2009;7(3-4):49-56. Persian

8. Bahri-Binabaj N, Moghiman M, Atarbashi M, Garche M. The survey of relationship between job burnout and health status of midwives and nurses. Ofogh-e-Danesh. 2003;9(1):99-104. Persian

9. Mohammadi S. Burnout and psychological health in high school teachers. J Iran Psychol. 2006;3(9):15-23. Persian

10. Anisi J, Eskandari M, Ghorbani Z, Abdolmohammadi E. Personal and organizational factors related to job burnout in naval personnel. J Behav Sci. 2010;4(3):249-53. Persian

11. Barrett L, Yates P. Oncology/haematology nurses: a study of job satisfaction, burnout, and intention to leave the specialty. Aust Health Rev 2002;25(3):109-21. [DOI]

12. Dick MJ. Burnout in nurse faculty: Relationships with management style, collegial support, and work load in collegiate programs. J Prof Nurs. 1986;2(4):252-60. [DOI]

13. Kouranian F, Khosravi AR, Esmaeili HE. The relationship between hardiness/locus of control and burnout in nurses. Ofoghe-e-Danesh. 2008;14(1):58-66. Persian

14. Rahmani F, Behshid M, Zamanzadeh V, Rahmani F. Relationship between general health, occupational stress and burnout in critical care nurses of Tabriz teaching hospitals. Iran $J$ Nurs. 2010;23(66):54-63. Persian

15. Lopez Franco M, Rodriguez Nunez A, Fernandez Sanmartin M, Marcos Alonso S, Martinon Torres F, Martinon Sanchez JM. [Burnout syndrome among health workers in pediatrics]. An Pediatr (Barc). 2005;62(3):248-51. [DOI]

16. Maslach C, Jackson S. Burnout in health professions: A social Psychological Analysis. 1982. Citado en Moreno Jiménez, B y Peñacoba Puente, C Facultad de Psicología Universidad Autónoma de Madrid. 1995.

17. Tummers GE, Landeweerd JA, van Merode GG. Organization, work and work reactions: a study of the relationship between organizational aspects of nursing and nurses' work characteristics and work reactions. Scand J Car Sci. 2002;16(1):52-8. [DOI] 
18. Koivula M, Paunonen M, Laippala P. Burnout among nursing staff in two Finnish hospitals. J Nurs Manag. 2000;8(3):149-58. [DOI]

19. Canadas-De la Fuente GA, Vargas C, San Luis C, García I, Cañadas GR, Emilia I. Risk factors and prevalence of burnout syndrome in the nursing profession. Int J Nurs Stud. 2015;52(1):240-9. [DOI]

20. Rafii F, Shamsikhani S, Zarei M, Haghani M. Burnout and its Relationship with the Nurses' Characteristics. Iran J Nurs. 2012;25(78):23-33. Persian
21. Frade MM, Vinagre GR, Zaragoza GI, Viñas SS, Antúnez ME, Alvarez GS, et al. [Burnout syndrome in different intensive care units]. Enferm Intensiva. 2008;20(4):131-40.

22. Santana Cabrera L, Hernandez Medina E, Eugenio Robaina P, Sanchez-Palacios M, Perez Sanchez R, Falcon Moreno R. [Burnout syndrome among nurses and nurses' aides in an intensive care unit and admission wards]. Enferm Clin. 2009;19(1):31-4. [DOI]

23. Adali E, Priami M. Burnout among nurses in intensive care units, internal medicine wards and emergency departments in Greek hospitals. ICUs Nurs Web J. 2002;11:1-19. 